\title{
Erratum to: Averaging generalized scalar field cosmologies I: locally rotationally symmetric Bianchi III and open Friedmann-Lemaître-Robertson-Walker models
}

\author{
Genly Leon $^{1, \mathrm{a}}{ }_{\mathbb{D}}$, Esteban González ${ }^{2, \mathrm{~b}}$, Samuel Lepe ${ }^{3, \mathrm{c}}$, Claudio Michea ${ }^{1, \mathrm{~d}}$, Alfredo D. Millano ${ }^{1, \mathrm{e}}$ \\ ${ }^{1}$ Departamento de Matemáticas, Universidad Católica del Norte, Avda. Angamos 0610, Casilla 1280, Antofagasta, Chile \\ 2 Departamento de Física, Universidad de Santiago de Chile, Avenida Ecuador 3493, Santiago, Chile \\ ${ }^{3}$ Instituto de Física, Facultad de Ciencias, Pontificia Universidad Católica de Valparaíso, Av. Brasil 2950, Valparaiso, Chile
}

Published online: 13 December 2021

(C) The Author(s) 2021

\section{Erratum to: Eur. Phys. J. C (2021) 81:414 https://doi.org/10.1140/epjc/s10052-021-09185-7}

In this article the following acknowledgment was replaced:

This research was funded by Agencia Nacional de Investigación y Desarrollo-ANID through the program FONDECYT Iniciación grant no. 11180126 and by Vicerrectoría de Investigación y Desarrollo Tecnológico at Universidad Católica del Norte. Ellen de los Milagros Fernández Flores is acknowledged for proofreading this manuscript and improving the English. We thank anonymous referee for his/her comments which have helped us improve our work.

The correct acknowledgment reads as follows:

The research of Genly Leon and Esteban González was funded by Agencia Nacional de Investigación y DesarrolloANID through the program FONDECYT Iniciación grant no. 11180126. Alfredo D. Millano was supported by Agencia Nacional de Investigación y Desarrollo - ANID - Subdirección de Capital Humano/Doctorado Nacional/año 2020folio 21200837. Claudio Michea was supported by Agencia Nacional de Investigación y Desarrollo - ANID - Subdirección de Capital Humano/Doctorado Nacional/año 2021 - folio
21211604. Vicerrectoría de Investigación y Desarrollo Tecnológico at Universidad Católica del Norte is anknowleged by finacial support. Ellen de los Milagros Fernández Flores is acknowledged for proofreading this manuscript and improving the English. We thank anonymous referee for his/her comments which have helped us improve our work.

The original article has been corrected.

Open Access This article is licensed under a Creative Commons Attribution 4.0 International License, which permits use, sharing, adaptation, distribution and reproduction in any medium or format, as long as you give appropriate credit to the original author(s) and the source, provide a link to the Creative Commons licence, and indicate if changes were made. The images or other third party material in this article are included in the article's Creative Commons licence, unless indicated otherwise in a credit line to the material. If material is not included in the article's Creative Commons licence and your intended use is not permitted by statutory regulation or exceeds the permitted use, you will need to obtain permission directly from the copyright holder. To view a copy of this licence, visit http://creativecomm ons.org/licenses/by/4.0/.

Funded by SCOAP $^{3}$.

The original article can be found online at https://doi.org/10.1140/ epjc/s10052-021-09185-7.

\footnotetext{
a e-mail: genly.leon@ucn.cl (corresponding author)

b e-mail: esteban.gonzalezb@usach.cl

c e-mail: samuel.lepe@pucv.cl

d e-mail: claudio.ramirez@ce.ucn.cl

e e-mail: alfredo.millano@alumnos.ucn.cl
} 